\title{
Involvement of follicular basement membrane and vascular endothelium in blood-follicle barrier formation of mice revealed by 'in vivo cryotechnique'
}

\author{
Hong Zhou ${ }^{1,2}$, Nobuhiko Ohno ${ }^{1}$, Nobuo Terada ${ }^{1}$, Sei Saitoh ${ }^{1}$, Yasuhisa Fujii ${ }^{1}$ and Shinichi Ohno ${ }^{1}$ \\ ${ }^{1}$ Department of Anatomy, Interdisciplinary Graduate School of Medicine and Engineering, University of Yamanashi, \\ 1110 Shimokato, Chuo-City, Yamanashi 409-3898, Japan and ${ }^{2}$ Department of Child, Adolescent and Women's \\ Health, School of Public Health, Peking University, Beijing 100083, China
}

Correspondence should be addressed to S Ohno; Email: sohno@yamanashi.ac.jp

\begin{abstract}
The molecular sieve with size- and charge selectivity in ovarian follicles, the so-called blood-follicle barrier (BFB), was examined during follicular development under physiological conditions to reveal ovarian structures responsible for the BFB by using our 'in vivo cryotechnique' (IVCT). Mouse ovary specimens were prepared with different methods including IVCT, immersion, or perfusion chemical fixation and quick-freezing following resection or perfusion. Their paraffin sections or cryosections were stained with hematoxylin-eosin or immunostained for serum proteins with different molecular weights: albumin, immunoglobulin (Ig) G1 heavy chain, inter- $\alpha$-trypsin inhibitor (I $\alpha$ l), fibrinogen, and IgM. Their immunoreactivity was better preserved with IVCT. The immunostaining for albumin was clearly observed in blood vessels, interstitium, and developing follicles, but that of IgG1, I $\alpha \mathrm{l}$, or fibrinogen was significantly decreased inside the follicles. IgM was immunohistochemically decreased throughout the interstitium outside blood vessels. The immunoreactivities of $\operatorname{lgG} 1$ and $\lg M$, as compared with albumin, were clearly changed along follicular basement membranes and around vascular endothelial cells respectively. These findings indicate that BFB functions throughout follicular development, and the follicular basement membrane and the vascular endothelium could play some significant roles in the permselectivity for such soluble proteins with intermediate and high molecular weight respectively. Reproduction (2007) 134 307-317
\end{abstract}

\section{Introduction}

Ovarian follicles of living animals have been known to be dynamic structures that undergo a gradual increase in volume during the various phases preceding ovulation, and the follicular development is always accompanied by significant structural changes of perifollicular capillary networks (Macchiarelli 2000). The perifollicular capillary networks are initially formed when the membrana granulosa of secondary follicles has developed into a multilayered follicle cell population. Furthermore, they also develop a single-layered to multilayered thick theca interna, as the secondary follicles grow up to larger or mature Graafian follicles (Kanzaki et al. 1982, Kitai et al. 1985, Kranzfelder \& Maurer-Schultze 1989, Yamada etal. 1994, Macchiarelli 2000, Jiang et al. 2002). These morphological changes of the follicular microvasculature have shown an urgent need for increased blood supply and nutritional support systems during the follicular development and oocyte maturation. In living animal ovaries, follicular fluid, which contributes to a microenvironment within the avascular compartment of follicles, is usually produced not only by secretion from the membrana granulosa cells but also by serum protein diffusion from the thecal capillaries during the follicle development (Donahue \& Stern 1968, Shalgi et al. 1973, Andersen et al. 1976). In the last year, a proteomic analysis revealed that a large number of 210 proteins in the human follicular fluid, including transferrin, ceruloplasmin, afamin, hemopexin, haptoglobin, and plasma amyloid protein, were acute inflammatory phase proteins, which actually supports a hypothesis that the common ovulation is comparable to an inflammatory event (Angelucci et al. 2006). They also identified a number of important antioxidant enzymes, including catalase, superoxide dismutase, glutathione transferase, paraoxonase, heat shock protein 27 , and protein disulfide isomerase, which probably protect the follicle maturation from various toxic injuries and oxidative stresses. Therefore, the follicular fluid is now assumed to nearly resemble the blood plasma, thereby providing the 
developing oocytes with the microenvironment that contains some necessary endocrine and non-endocrine growth factors (Angelucci et al. 2006). However, several earlier studies have already described definite differences in both protein compositions and concentrations between the follicular fluid and the blood serum, which led to a proposal of the blood-follicle barrier (BFB; Zachariae 1958, Shalgi et al. 1973). They have described the BFB as a 'molecular sieve' with size selectivity consisting of the capillary endothelium, the subendothelial basal lamina, the theca interna, the follicular basement membrane, and the membrana granulosa; the BFB allowed low-molecular weight proteins $(500 \mathrm{kDa})$ to enter the follicular fluid, but blocked the entrance of higher molecular components or larger particles, such as colloidal gold (1000 kDa) (Shalgi et al. 1973, Cran et al. 1976). Some subsequent studies have also revealed another charge selectivity and involvement of nitric oxide in the functional mechanism of BFB, and so putative significance of BFB was also proposed (Powers et al. 1995, Hess et al. 1998, Zhuo \& Kimata 2001). However, in spite of such accumulating pieces of evidence about the BFB properties, morphological data of BFB under physiological conditions in vivo are still limited, because the conventional morphological studies on BFB have been often made with animal models of artificially induced ovulation under experimental conditions. Furthermore, the significant structures within the follicles that contribute to the BFB during the routine follicular development still remain to be elucidated.

Although the morphology and molecular distribution of animal ovaries have been examined by various preparation techniques, each technique has some limitations for morphofunctional and immunohistochemical analyses of cells and tissues. Among them, the conventional preparation procedures, such as perfusion- or immersion fixation (IM) with chemical fixatives, often produce morphological artifacts caused by anoxia of tissue resection, artificial perfusion pressures with fixatives, and dehydration shrinkage with organic solvents (Kellenberger 1991, Hippe-Sanwald 1993, Shiurba 2001, Li et al. 2005, Ohno et al. 2006, Zhou et al. 2007). For overcoming these problems, our 'in vivo cryotechnique' (IVCT) has been developed since 1996 to directly clarify functioning morphology and immunodistribution of molecular components in cells and tissues of living animal organs (Ohno et al. 1996, Terada et al. 2006a). The IVCT is a kind of cryofixation method in which target organs of living animals are directly frozen in vivo without tissue resection or perfusion fixation (PF), thus enabling us to capture transient changes of tissue and cell morphology under different hemodynamic conditions and also molecular changes such as rapid phosphorylation of signaling molecules (Ohno et al. 2005a, Terada et al. 2006b). In addition, it was already reported with our several studies that the IVCT followed by freeze-substitution very efficiently retained soluble extra- and intracellular molecules within tissues and cells, and their clear localizations or dynamic structural changes in vivo were histochemically or immunohistochemically revealed on paraffin-embedded sections (Zea-Aragon et al. 2004, Terada et al. 2005, Li et al. 2006a, Liao et al. 2006, Ohno et al. 2006).

In the present study, we have morphologically and immunohistochemically examined living mouse ovaries by the IVCT and focused on the actual clarification of their BFB under normal blood circulation. As the immunolocalizations of different serum proteins in blood vessels, interstitium, and ovarian follicles could be clearly detected with the IVCT, the BFB appeared to exist from early stages of normal folliculogenesis in the living mouse ovaries. Also, the follicular basement membranes in addition to the vascular endothelium were suggested to play some significant roles in the formation of BFB against serum proteins, depending partly on their molecular sizes.

\section{Material and Methods}

\section{Tissue preparation methods}

All animal experiments were performed in accordance with the guidelines by the Animal Care and Use Committee, University of Yamanashi. Each cycle of all mice was checked by the routine vaginal smear examination, and the selected mice at proestrus to estrus stages were always used for further analyses. Ovarian tissues of 20 C57BL/6 mice aged 8-9 weeks, which were anesthetized with inhalation of diethyl ether, were prepared by the following different methods. i) IM and alcohol dehydration (IM-DH): the ovarian tissues of two mice were surgically resected and immersed in $0.1 \mathrm{M}$ phosphate buffer solution containing $2 \%$ paraformaldehyde (PFA; $\mathrm{pH} 7.4$ ) overnight. ii) $\mathrm{PF}-\mathrm{DH}$ : two mice were transcardially perfused with the similar buffered $2 \%$ PFA, and their ovarian tissues were resected and immersed in the same fixative overnight. iii) Conventional quickfreezing for fresh resected tissues (FQF): the ovarian tissues were surgically removed from two mice and quickly frozen by plunging into the isopentane-propane cryogen $\left(-193^{\circ} \mathrm{C}\right)$, as described before (Ohno et al. 2004). iv) PF-QF: two mice were transcardially perfused with the buffered 2\% PFA as described above, and their ovarian tissues were resected and quickly frozen as in FQF. v) IVCT: the isopentane-propane cryogen was poured over the carefully exposed ovaries of 12 mice, as reported before (Ohno et al. 2006), and then the completely frozen tissues were removed with a dental electric drill in liquid nitrogen $\left(-196^{\circ} \mathrm{C}\right)$. All the specimens prepared by the methods $(\mathrm{i}-\mathrm{v})$ were additionally treated with the conventional $\mathrm{DH}$ (i and ii) or the routine freeze-substitution with $2 \%$ PFA (iii-v) and embedded in either paraffin wax or optimal cutting 
temperature (OCT) compound, as described previously (Ohno et al. 2004, Terada et al. 2005). Briefly, in the freeze-substitution, freeze-substitution solution was first prepared by adding $20 \%(\mathrm{w} / \mathrm{v})$ PFA to pure acetone to finally become $2 \%$ PFA, followed by complete dehydration with sufficient amounts of Molecular Sieves 3A (Nacalai Tesque, Kyoto, Japan). The frozen ovarian specimens were incubated in the PFA-acetone solution cooled down at about $-80^{\circ} \mathrm{C}$ in dry ice-acetone for $48 \mathrm{~h}$, and gradually warmed up to room temperature. Thereafter, the specimens were either infiltrated with xylene for the common paraffin embedding or rehydrated with PBS containing 30\% sucrose for embedding in OCT compound.

\section{Immunohistochemistry of serum proteins}

All paraffin-embedded samples were cut at $3 \mu \mathrm{m}$ thickness and mounted on glass slides treated with 3-aminopropyltriethoxysilane (Nacalai Tesque). The cut sections were then deparaffinized with xylene and a graded series of ethanol. For histological analyses, some serial sections were routinely stained with hematoxylineosin (HE). For immunohistochemical analyses, others were incubated with $1 \%$ hydrogen peroxide in the PBS for $1 \mathrm{~h}$ and then 5\% normal rabbit serum (NRS) in PBS for $1 \mathrm{~h}$. They were immunostained with various primary antibodies in PBS containing $5 \%$ NRS at $4{ }^{\circ} \mathrm{C}$ overnight. The primary antibodies were as follows: goat anti-mouse albumin antibody at dilution of 1:5000, goat anti-mouse immunoglobulin (Ig) G1 heavy chain antibody at dilution of 1:500, and goat anti-mouse IgM antibody at dilution of 1:500. They were all purchased from Bethyl Laboratories (Montgomery, TX, USA). The immunostained sections were then incubated in biotin-conjugated rabbit anti-goat IgG (Vector Laboratories, Burlingame, CA, USA) at room temperature for $1 \mathrm{~h}$. The immunoreaction products were visualized with Vectastain $A B C$ reagent (Vector Laboratories) and metal-enhanced DAB substrate kit (Pierce, Rockford, IL, USA), and additionally fixed with $0.04 \%$ osmium tetroxide solution as described before (Ohno et al. 2005b). All immunostained sections were counterstained with methylgreen, embedded in glycerol, and observed with a light microscope (BX-61; Olympus, Tokyo, Japan).

For the double immunolabeling study, cryosections at $6 \mu \mathrm{m}$ thickness and also deparaffinized sections on the glass slides were blocked with $2 \%$ gelatin (Sigma) in PBS for $1 \mathrm{~h}$ and immunostained with the primary antibodies at $4{ }^{\circ} \mathrm{C}$ overnight as follows: i) goat anti-mouse albumin antibody at dilution of 1:4000 and rabbit anti-human fibrinogen antibody (Dako Japan, Kyoto, Japan) at dilution of 1:500 or rabbit anti-human inter- $\alpha$-trypsin inhibitor (Ial) antibody (Dako Japan) at dilution of 1:400; and ii) goat anti-mouse type IV collagen antibody (Southern Biotechnology Associates, Birmingham, AL, USA) at dilution of 1:50 and rabbit anti-mouse albumin antibody (Bethyl Laboratories) at dilution of 1:4000 or rabbit anti-mouse IgGfc antibody (Bethyl Laboratories) at dilution of 1:500. They were then incubated with donkey anti-rabbit $\operatorname{lgG}$ antibody coupled with Alexa Fluor 488 at dilution of 1:400, donkey anti-goat IgG antibody coupled with Alexa Fluor 546 at dilution of 1:400, and To-pro-3 at dilution of 1:500 (Invitrogen) with $2 \%$ gelatin in PBS at room temperature for $1 \mathrm{~h}$. The immunostained cryosections were embedded in Vectashield (Vector Laboratories) and observed with a confocal laser scanning microscope (FV1000; Olympus).

\section{Double fluorescence labeling with periodic acid-Schiff (PAS) and immunostaining}

We used the fluorescence emission of PAS staining for fluorescent detection of basement membranes around blood vessels because the PAS-fluorescence emission could be most effective to visualize the PAS-stained glomerular basement membrane (Li et al. 2006b). First, $3 \mu \mathrm{m}$ thick deparaffinized sections were routinely stained for PAS, as described previously ( $\mathrm{Li}$ et al. 2006b). Subsequently, all the sections were incubated with $5 \%$ gelatin in PBS for $1 \mathrm{~h}$ and then with the goat anti-mouse $\lg M$ antibody at $4{ }^{\circ} \mathrm{C}$ overnight. They were then incubated with the donkey anti-goat IgG antibody coupled with Alexa Fluor 488 and To-pro-3 for $1 \mathrm{~h}$, and embedded in Vectashield. All sections stained with PAS reaction were examined with FV1000 (Olympus), as described before (Li et al. 2006b).

\section{Results}

\section{Immunolocalization of albumin in ovarian follicles}

We have first examined the immunolocalization of albumin (Fig. 1b, d, f, h and j) in ovarian tissues prepared with IVCT (Fig. 1a and b), FQF (Fig. 1c and d), PF-DH (Fig. 1e and f), IM-DH (Fig. 1g and h), and PF-QF (Fig. 1i and $\mathrm{j}$ ) on the serial sections, corresponding to the areas with HE staining (Fig. 1a, C, e, g and i). In the specimens prepared with IVCT, immunoreactivity of albumin was detected in blood vessels (Fig. 1a and b, arrowheads), interstitium (Fig. 1b, thin arrows), and ovarian follicles (Fig. 1b, thick arrows). Immunostaining for albumin was also observed in zona pellucida around the oocyte. The immunolocalization of albumin observed with IVCT was similar to that with FQF (Fig. 1c and d); albumin was clearly immunolocalized in both interstitium (Fig. 1d, thin arrow) and follicles (Fig. 1d, thick arrows) in addition to blood vessels. To the contrary, in the specimens prepared with PF-DH (Fig. 1e and f) or IM-DH (Fig. 1g and h), less immunoreactivity of albumin was detected inside the ovarian follicles (Fig. $1 \mathrm{f}$ and $\mathrm{h}$, thick arrows), although previous biochemical studies have already reported large amounts of albumin in the follicular fluid (Shalgi et al. 1973, Collins et al. 1997). Moreover, by PF-QF, some diffuse immunostaining for 
HE
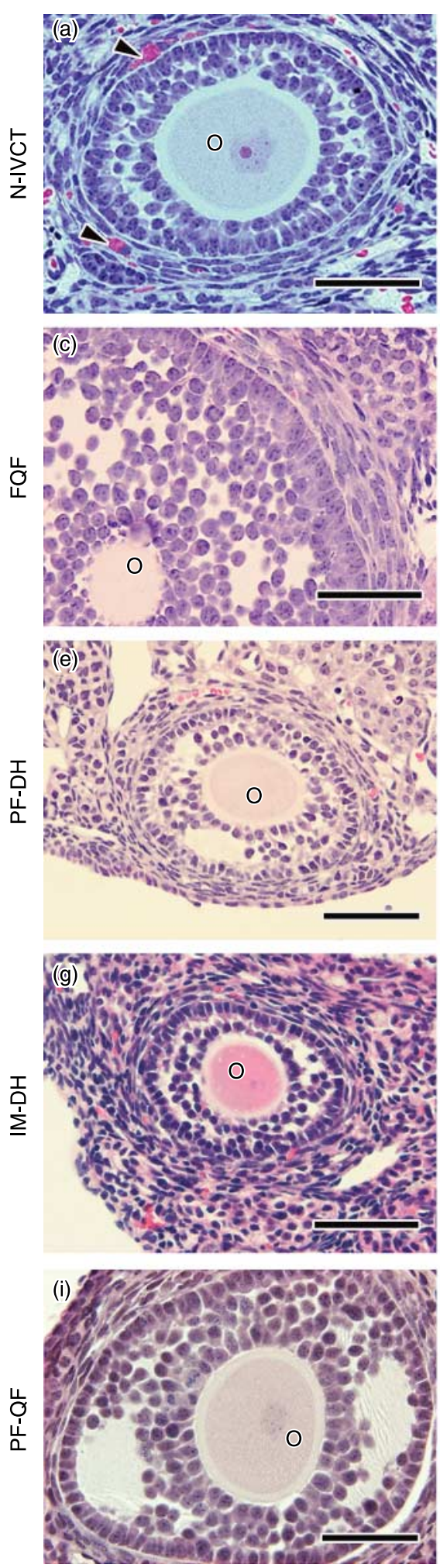

Alb
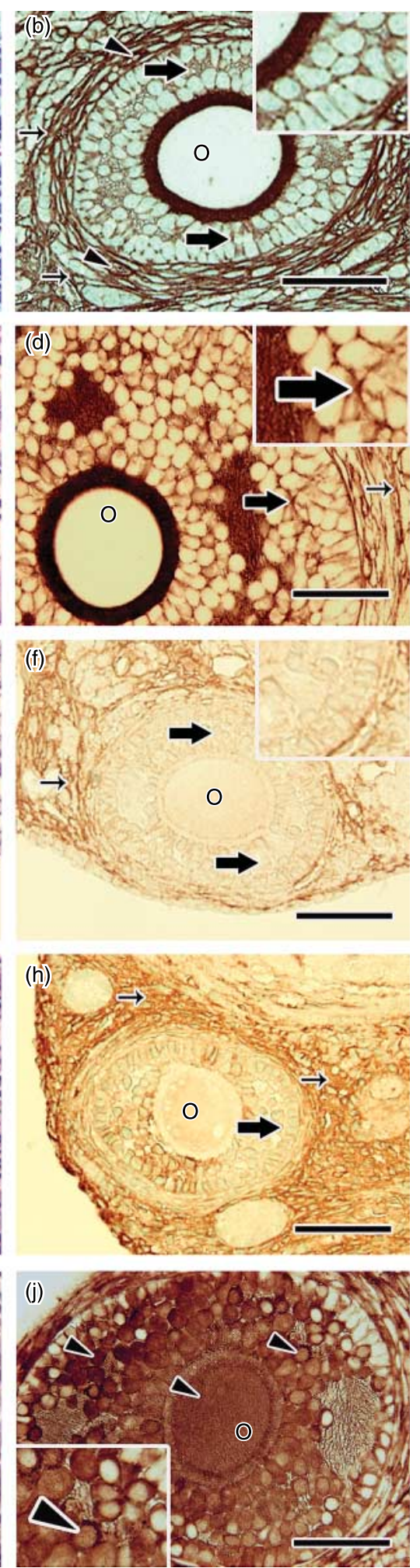

Figure 1 Light micrographs of hematoxylin-eosin (HE) staining (a, c, e, g and i) and immunostaining for serum albumin (Alb; b, d, f, h and j) on serial sections prepared by in vivo cryotechnique under normal circulation (N-IVCT, a and b), quickfreezing of resected fresh tissues (FQF, c and d), perfusion fixation (PF-DH, e and $\mathrm{f}$ ) or immersion fixation (IM-DH, g and h) followed by alcohol dehydration and perfusion fixation followed by quick-freezing (PF-QF, i and j). ( $a$ and $b$ ) In the specimens prepared by N-IVCT, the immunoreactivity of albumin is detected in blood vessels pointed with black arrowheads in (a) (b, arrowheads), interstitium (b, thin arrows), and ovarian follicles (b, thick arrows). Immunostaining is also observed in zona pellucida. (c-h) The immunolocalization is similar to that in the specimens prepared by FQF (d, thin or thick arrows). In the specimens prepared by PF-DH (e and f) or IM-DH ( $\mathrm{g}$ and $\mathrm{h}$ ), the immunoreactivity of albumin decreases inside the ovarian follicles ( $f$ and $h$, thick arrows), as compared with that in the interstitium ( $f$ and $h$, thin arrows). ( $i$ and $j$ ) By PF-QF, diffuse immunostaining for albumin can be detected inside an oocyte and some granulosa cells (j, arrowheads). O, oocytes. Bars, $50 \mu \mathrm{m}$. 
albumin could be detected not only in extracellular spaces but also inside cytoplasm of some oocytes and granulosa cells (Fig. 1j, arrowheads). We also examined immunolocalizations of the other serum proteins including $\operatorname{lgG} 1$ and $\operatorname{IgM}$ and found their decreased immunoreactivity in PF-DH and IM-DH or similar immunolocalization in PF-QF (data not shown). These findings indicated that the immunodistribution of serum proteins in vivo were significantly affected during preparation procedures of organ perfusion or chemical fixation followed by $\mathrm{DH}$, and the IVCT would be more useful for immunohistochemical analyses of soluble serum proteins in living mouse ovaries.

\section{Immunohistochemical examination of BFB in com- bination with IVCT}

Next, serial paraffin sections prepared with IVCT were stained with $\mathrm{HE}$ or immunostained for three serum proteins including albumin with about $69 \mathrm{kDa}$ molecular weight, IgG1 with about $150 \mathrm{kDa}$ molecular weight, and IgM with about 900 kDa molecular weight, and their immunoreactivities were compared around follicles in different stages. In the present study, the following follicular classification was used: the primordial follicle is composed of a primary oocyte and a single layer of flat follicular cells surrounding the oocyte; the early primary follicle displays a single layer of cuboidal follicular cells that surround the primary oocyte; the late primary follicle displays a stratified layer around the oocyte; the secondary or antral follicle shows more layers of follicular cells, and some antrum appears in the intercellular spaces; the mature or Graafian follicle is characterized by the presence of a large fluid-filled antrum (Ross et al. 1989, Gartner \& Hiatt 1994). In a late primary follicle on the serial sections (Fig. 2), all three proteins were clearly immunolocalized in blood vessels (Fig. 2, white arrowheads). In addition, immunostainings for both albumin (Fig. 2b, black arrowhead) and IgG1 (Fig. 2c, black arrowhead) were detected in the interstitium, but IgG1 alone was more weakly immunostained inside the primary follicle (Fig. 2c, black arrow). To the contrary, immunostaining for IgM was decreased in follicles, interstitium, and theca layers, and was hardly detected along the zona pellucida (Fig. 2d, white arrow), although it was strongly observed inside blood vessels of the theca layers (Fig. 2d, white arrowheads). Double fluorescence labeling of IgM with PAS-positive basement membranes around blood vessels is shown in Fig. 6. From these findings, it is suggested that the albumin can easily enter ovarian follicles, but either IgG1 or IgM was blocked around the follicles and the blood vessels respectively.

We also examined these immunohistochemical findings of serum proteins in other stable or developing follicles, such as primordial or antral ones (Fig. 3a-h). The similar immunoreactivity of three serum proteins was observed inside blood vessels around those follicles (Fig. 3a-h, white arrowheads). The albumin immunostaining was detected in them (Fig. $3 b$ and $f$, black arrows). Moreover, immunostainings for IgG1 and IgM were weakly detected inside those follicles (Fig. 3c, d, g and $h$, black arrows), and also that for IgM was decreased in the theca layers and interstitium (Fig. $3 \mathrm{~d}$ and $\mathrm{h}$, black arrowhead), as inside or around the primordial or antral follicles. To the contrary, the stronger immunoreactivity of the three serum proteins was usually observed in the ovulated follicles (Fig. 4a-d), and no significant difference of their immunoreactivity was observed among blood vessels, interstitium, and follicles. This
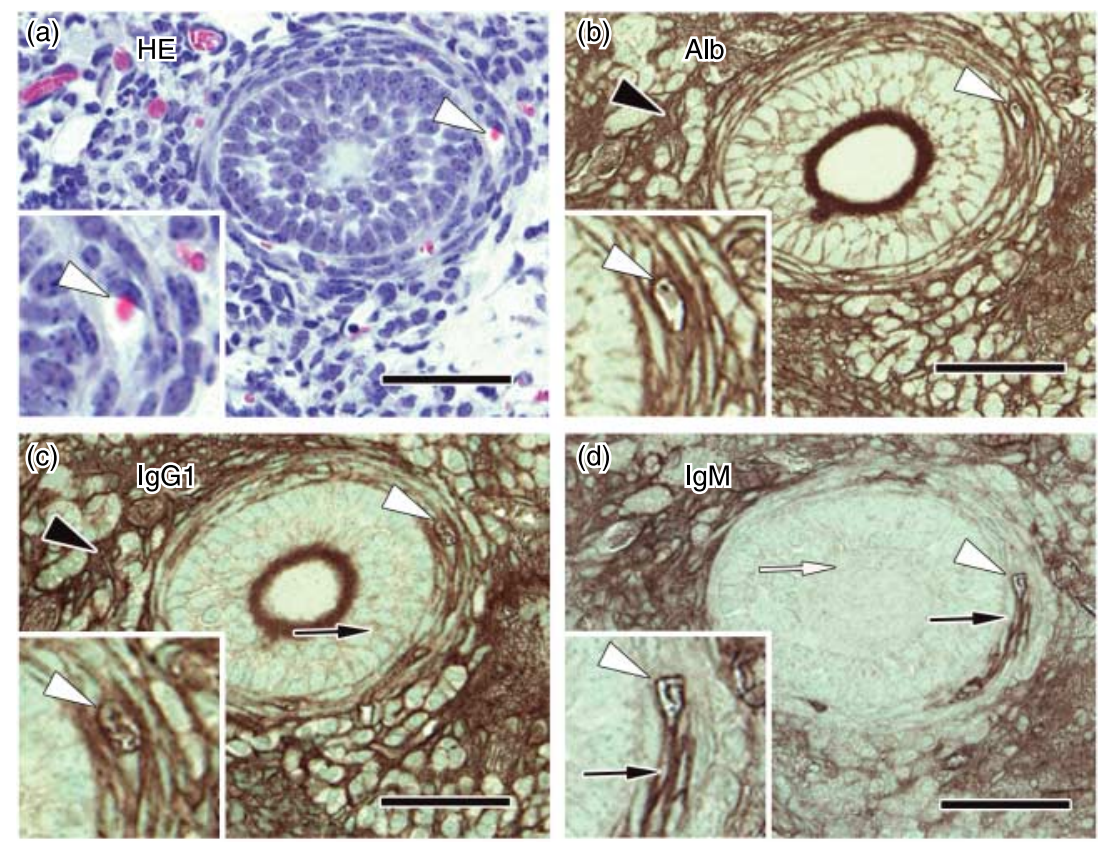

Figure 2 Serial paraffin sections of late primary follicle in living mouse ovaries prepared by in vivo cryotechnique and stained with hematoxylineosin $(\mathrm{HE}, \mathrm{a})$ or immunostained for albumin (Alb, b), immunoglobulin G1 (IgG1, c), or IgM (d). The areas around blood vessels are twice magnified in the insets. In the late primary follicle, multiple layers of follicular cells surround the oocyte. The strong immunoreactivity of three serum proteins is clearly detected inside blood vessels ( $a-d$, white arrowheads). Although the immunostainings for albumin (b, black arrowhead) and IgG1 (c, black arrowhead) are clearly detected in the theca layers and interstitium of ovary, that for IgG1 is weakly seen inside ovarian follicles (c, black arrow). Another immunostaining for IgM is decreased in the interstitium and theca layers (d, black arrows) and hardly detected in the follicles (d, white arrow), although it is strongly observed inside the blood vessels of theca layers (d, white arrowheads). Bars, $50 \mu \mathrm{m}$. 

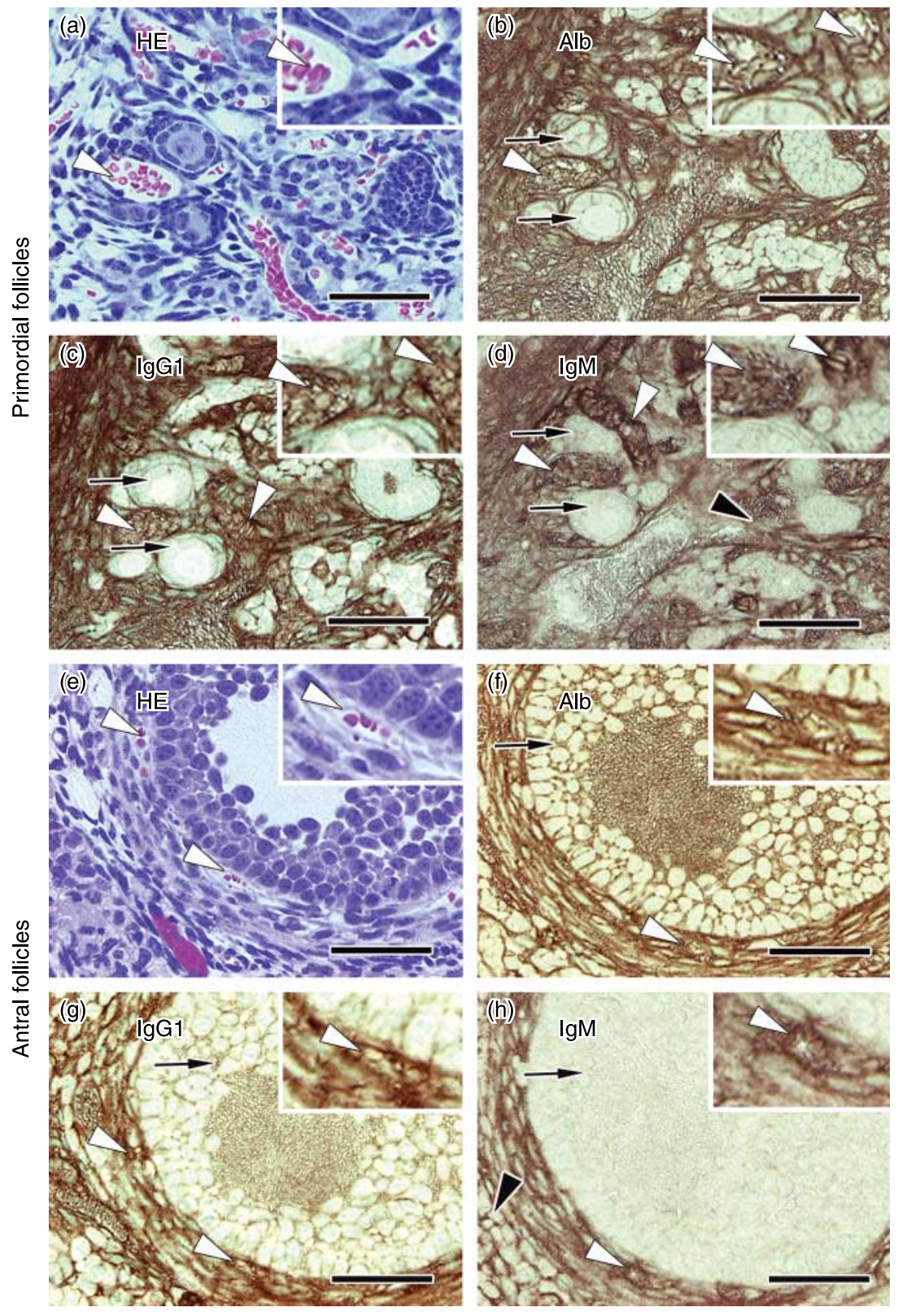

Figure 3 Serial paraffin sections of primordial (ad) and antral follicles (e-h) in living mouse ovaries prepared by in vivo cryotechnique and stained with hematoxylin-eosin ( $\mathrm{HE}$, a and e) or immunostained for albumin (Alb, $b$ and $f$ ), immunoglobulin G1 (IgG1, c and g), or IgM (d and $h$ ). The areas around blood vessels are twice magnified in the insets. The primordial follicles (b-d, black arrows) are composed of a single layer of flat follicular cells surrounding the oocyte, and antral follicles have more layers of follicular cells and antrum among them. The strong immunoreactivities of three serum proteins are detected inside blood vessels $(\mathrm{a}-\mathrm{h}$, white arrowheads). The immunostainings for $\lg \mathrm{G} 1$ and IgM are weakly seen inside the follicles (c, d, g and $\mathrm{h}$, black arrows), and the IgM immunostaining is also decreased in the theca layers and interstitium ( $\mathrm{d}$ and $\mathrm{h}$, black arrowheads), as in or around the late primary follicles. Bars, $50 \mu \mathrm{m}$. possible destruction of BFB upon ovulation stimuli has been already reported in the animal ovulation model (Powers et al. 1995, Hess et al. 1998).

We also examined immunolocalizations of $|\alpha|$ (with 220 kDa molecular weight) or fibrinogen (with $340 \mathrm{kDa}$ molecular weight) in developing ovarian follicles on cryosections prepared with IVCT (Fig. 5), because previous studies indicated that distributions of serum proteins with middle-sized molecules were largely affected by BFB in mouse ovaries (Hess et al. 1998). In our findings, immunoreactivities of $|\alpha|$, fibrinogen, and albumin were clearly detected in the interstitium and theca layers of ovaries (Fig. 5a, b, d-h, arrows), but immunoreactivities of both $|\alpha|$ and fibrinogen were more weakly detected inside the ovarian follicles, as compared with the albumin immunoreactivity (Fig. 5a, $\mathrm{d}$, e and h, arrowheads).

We further examined the soluble and structural components crucial for BFB around blood vessels and ovarian follicles by using double immunofluorescence labeling of albumin (Fig. 6a, d and e) or IgGfC (Fig. 6f, i and j) and type IV collagen, a major structural component of basement membranes (Fig. 6b and g). We also performed another immunofluorescence labeling for IgM (Fig. 6k, n and o) with PAS-fluorescence emission (Fig. 6l, $\mathrm{n}$ and o) in living mouse ovaries prepared by IVCT. The PASfluorescence emission in the tissue specimens has been already used for the clear visualization of glomerular 

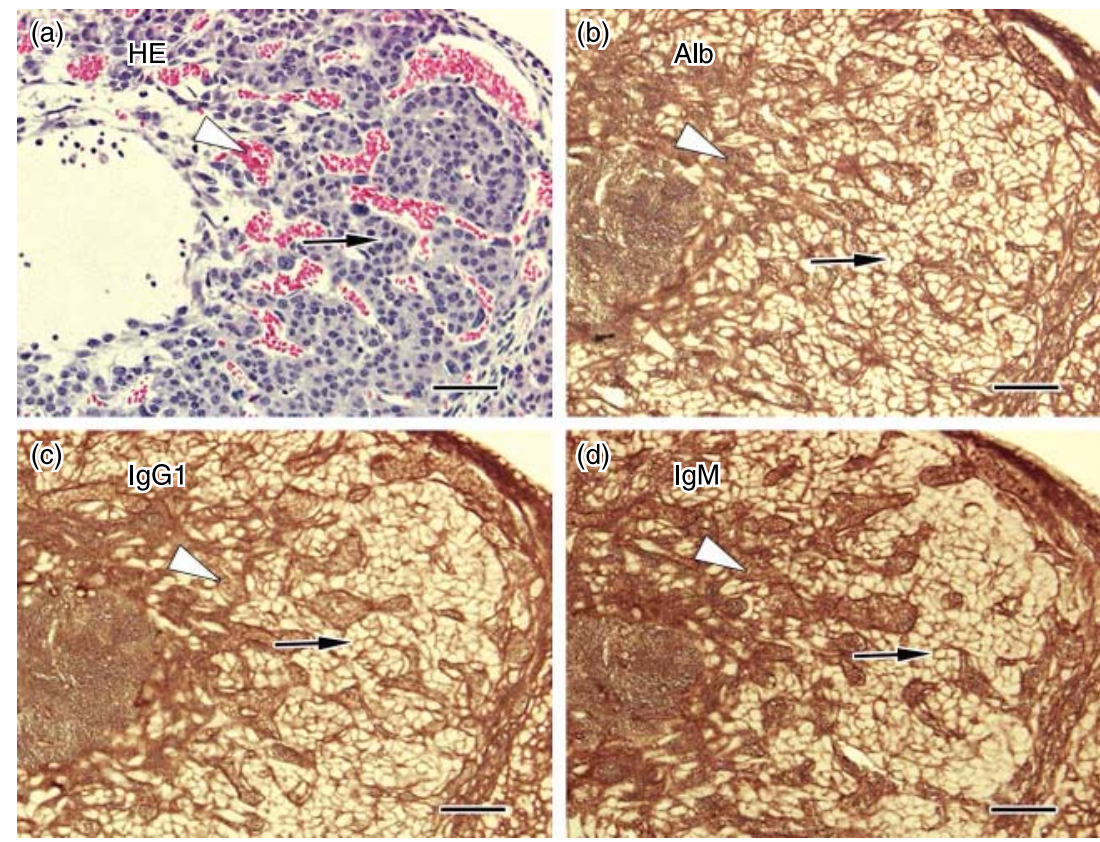

Figure 4 Serial paraffin sections of ovulated follicles in living mouse ovaries prepared by in vivo cryotechnique and stained with hematoxylin-eosin $(\mathrm{HE}, \mathrm{a})$ or immunostained for albumin (Alb, b), immunoglobulin G1 (IgG1, c), or $\lg M(d)$. The strong immunoreactivities of the three serum proteins are clearly detected not only inside the blood vessels (white arrowheads) but also outside the blood vessels in the ovulated follicles (black arrows). Bars, $50 \mu \mathrm{m}$. basement membranes of living mouse kidneys (Li et al. 2006b). The immunoreactivity of albumin in the ovarian follicles, theca layers, and interstitium was almost similarly detected in living mouse ovaries (Fig. 6a; black arrow and black arrowhead), which was bordered by the type IV collagen-immunopositive basement membrane (Fig. 6b, d and e, white arrowheads). Although another immunostaining for IgGfc was clearly detected in the theca layers and interstitium of living mouse ovaries (Fig. 6f; black arrow), it was more weakly detected inside ovarian follicles (Fig. 6f, i and $\mathrm{j}$, black arrowhead), and the basement membrane immunopositive for type IV collagen (Fig. 6f, g, i and j, white arrowheads) bordered the immunoreactivity changes of IgGfc (Fig. $6 f$ and i, black arrowheads). The immunoreactivity of IgM was detected exclusively in blood vessels around the theca interna (Fig. 6k and o, black arrowheads), but it was not detected in the ovarian follicles and interstitium (Fig. 6k, black arrow). At higher magnification, the immunolocalization of IgM was mostly restricted along endothelial cells of the blood vessels surrounded by PAS-positive basement membranes (Fig. 60; white arrowheads). From these findings, it is suggested that the BFB plays some selective functions throughout the follicular development until ovulation at the final stage of follicles. Furthermore, the in vivo permeability of large molecules, such as IgM, is blocked at the endothelial layers of blood vessels in living mouse ovaries, but the follicular basement membranes are playing some crucial roles for efficient inhibition in permselectivity, which means selectivity of molecular permeation, of middle-sized molecules, such as serum IgG.

\section{Discussion}

As initially described over 40 years ago, the BFB has remained to be a biological and clinical subject for
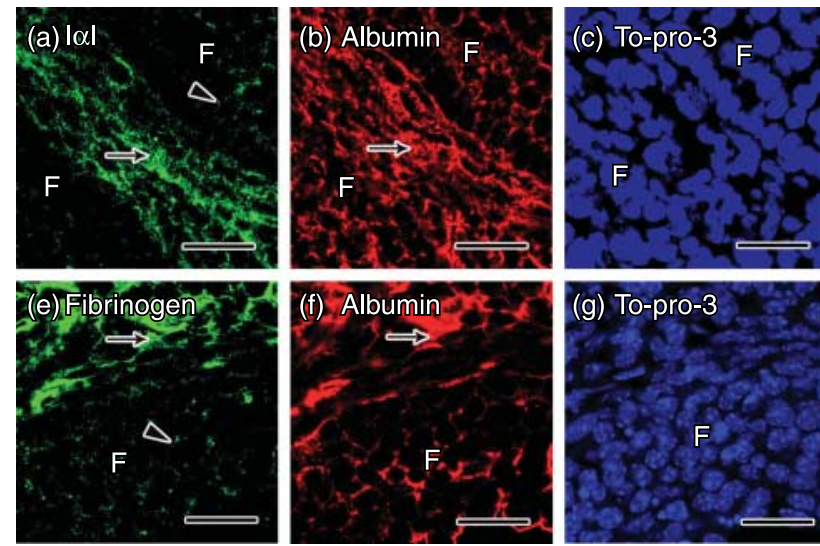

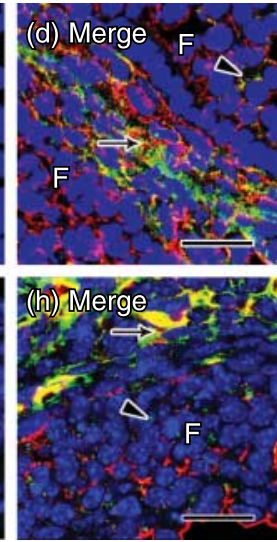

Figure 5 Double immunofluorescence labeling of albumin (b, d, f and $h$, red) and $|\alpha|$ (a and d, green) or fibrinogen (e and $h$, green) in antral follicles of living mouse ovaries on cryosections prepared with in vivo cryotechnique. The cellular nuclei are labeled with To-pro-3 (c, d, g and h, blue).

Although the immunostainings for lal, fibrinogen, and albumin are detected in the theca layers and interstitium of living mouse ovaries (a, b, d, e, f and $h$, arrows), the $|\alpha|$ and fibrinogen immunostainings are weakly seen inside the follicles, as compared with those for albumin $(\mathrm{a}, \mathrm{d}$, e and $\mathrm{h}$, arrowheads). F, ovarian follicle. Bars, $20 \mu \mathrm{m}$. 

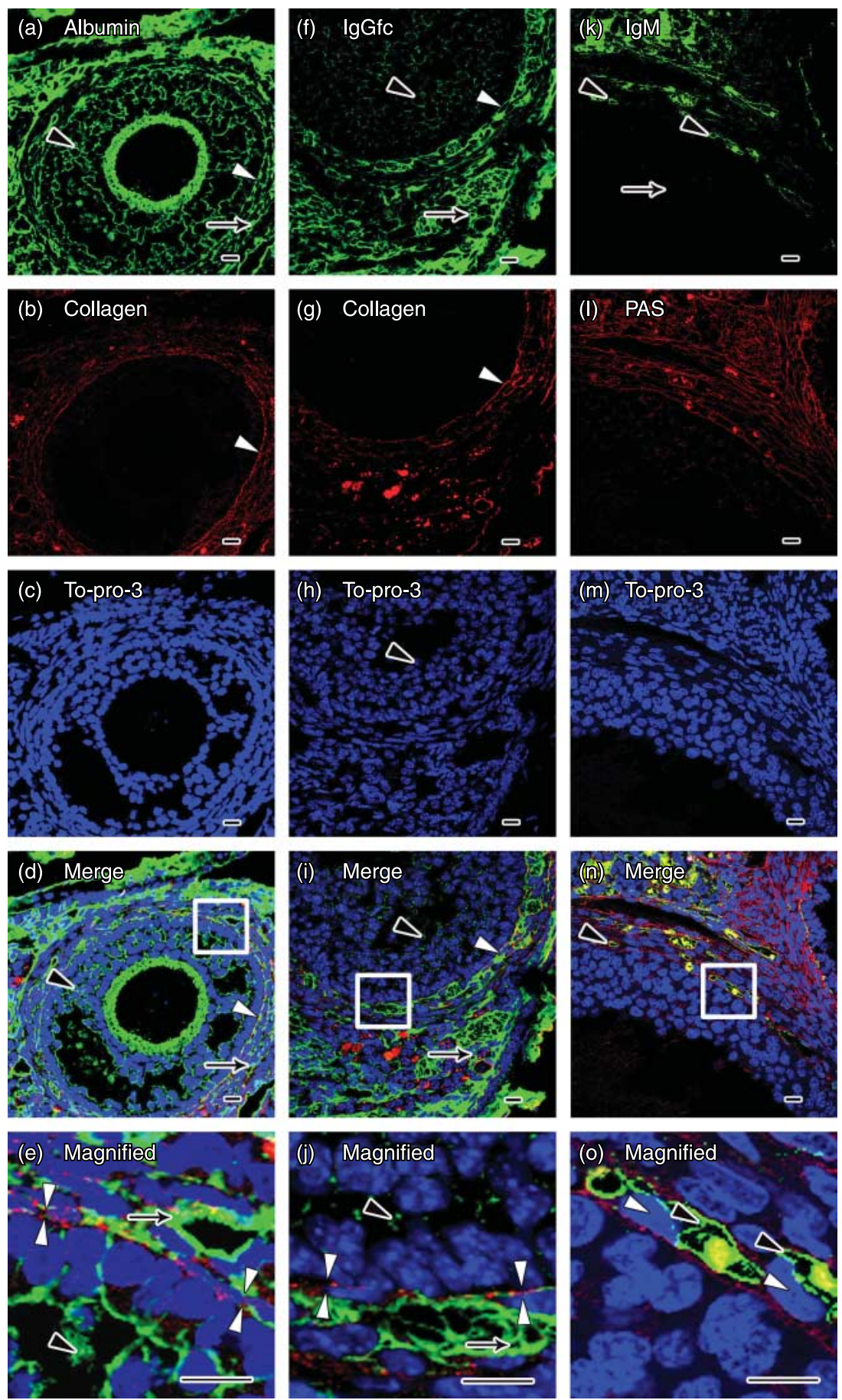

Figure 6 Double immunofluorescence labeling of type IV collagen (b, d, e, g, i and j, red) and albumin ( $a, d$ and e, green) or immunoglobulin $\mathrm{G}$ (IgGfC, $f$, i and j, green) and immunofluorescence staining for $\operatorname{lgM}(\mathrm{k}, \mathrm{n}$ and $\mathrm{o}$, green) with periodic acid-Schiff staining fluorescence emission (PAS, I, $\mathrm{n}$ and $\mathrm{o}$, red) around antral follicles of living mouse ovaries prepared by in vivo cryotechnique. The cellular nuclei are labeled with To-pro-3 (c-e, $\mathrm{h}-\mathrm{j}$ and $\mathrm{m}-\mathrm{O}$, blue), and the areas represented by rectangles in $(\mathrm{d}, \mathrm{i}$, and $\mathrm{n})$ are highly magnified in (e, j, and o) respectively. (a-e) The immunoreactivities of albumin are similarly detected inside the follicle (a, $d$ and e, black arrowheads) and in the theca layers and interstitium (a, d and e, black arrows). The border between them is marked by the basement membrane $(a, b, d$ and e, white arrowheads). ( $f-j)$ Although the immunostaining for IgGfC is clearly detected in the interstitium of ovaries ( $f$, i and $j$; black arrows), it is weakly detected inside the antral follicle $(f, i$ and $j$, black arrowheads). The basement membrane ( $f, g$, $i$ and $\mathrm{j}$, white arrowheads) borders the strong immunoreactivity of IgGfc. (k-O) The immunoreactivity of IgM is clearly seen in the blood vessels of theca interna ( $\mathrm{k}, \mathrm{n}$ and $\mathrm{o}$, black arrowheads), while it is not detected inside the antral follicle ( $k$, black arrow). The immunolocalization of IgM is mostly restricted inside the endothelial cells in the blood vessels surrounded by PAS-positive basement membranes (o, white arrowheads). Bars, $10 \mu \mathrm{m}$. developing ovarian follicles and some ovarian diseases. The microscopic analyses with non-physiological tracers, i.e. colloidal gold and Evans Blue dye, led to a suggestion that BFB acting like a molecular sieve was permeable only for exogenous proteins under $500 \mathrm{kDa}$ in molecular size (Cran et al. 1976). Later, almost 10 years ago, Hess et al. (1998) provided their physiological evidence for the actual existence and regulation mechanism of BFB in an animal ovulation model, indicating that the BFB would be charge- and size selective in living mouse ovaries. However, the detailed structural properties of BFB and also how it affects the passage of serum proteins in vivo have remained unclear in past decades. Although a number of biochemical and morphological studies have been reported to describe such BFB existence, the present study is the first to demonstrate immunohistochemically the in vivo permeability of BFB against endogenous serum proteins 
with different molecular sizes during follicular development and also the histological layers responsible for the actual barrier functions of BFB.

In our studies, serum proteins with low molecular sizes, such as albumin, were abundantly immunolocalized in ovarian follicles at different developing stages, but those with intermediate molecular sizes, such as $\operatorname{lgG} 1,|\alpha|$, and fibrinogen, were more weakly detected in the ovarian follicles. To the contrary, the high molecular weight of IgM was almost totally blocked at the basement membrane of the ovarian follicles. These molecular differences in immunolocalizations of serum proteins undoubtedly support a previous idea that the molecular sizes are responsible for the BFB selectivity (Hess et al. 1998). Additionally, in the present study, the follicular basement membrane of living mouse ovaries was found to play some functional roles in the permselectivity for serum proteins, such as $\operatorname{lgG} 1$, with the intermediate molecular sizes. On the other hand, endothelial cells of the blood vessels in ovaries were responsible for blocking the passage of serum proteins, such as IgM, with high molecular sizes. Since intensities of immunostaining for different molecules depend on nonlinear saturation of different antibodies, it should be noted that relative contribution of these different structures to BFB selectivity remained unclear in the present study. However, as different intensities of immunostaining on single histological sections depend on the amounts of antigens, the relative immunoreactivity difference of each molecule among various structures of ovary, observed on the single sections in the present study, suggested significant involvement of both follicular basement membranes and endothelial cells in BFB. It is generally accepted that endothelial cells of blood vessels in other organs usually control the passage of soluble serum components and circulating cells from the blood vessels to tissue parenchyma, and also that intercellular junctions closely mediate both adhesion and communication between adjoining endothelial cells (Bazzoni \& Dejana 2004, Orlova et al. 2006). The junctional complexes are generally composed of tight junctions, adherens junctions, and gap junctions, and the previous studies already showed that the tight junctions were more important for the strict control of endothelial permeability (Aijaz et al. 2006, Bazzoni 2006). The expression and organization of these junctional complexes usually depend on the type of blood vessels and the permeability requirements of functioning organs (Madara 1989). For instance, such intercellular junctions are well developed in the central nervous system, forming the widely known 'blood-brain barriers' (Wolburg \& Lippoldt 2002), whereas postcapillary venules, which allow dynamic trafficking of circulating cells and soluble proteins, often display poorly organized tight junctions (Dvorak et al. 1995). To the contrary, the intercellular communication between endothelial cells in living mouse ovaries is not well understood in terms of molecular permeability against soluble serum proteins. Although one previous study clarified the endothelial blockage of middle-sized serum proteins, which was controlled by nitric oxide in ovaries (Powers et al. 1995), the present study showed abundant immunolocalizations of such middle-sized serum proteins in the ovarian interstitium and indicated that the ovarian endothelial cells possess much less strict selectivity, which more efficiently blocks the serum proteins with large molecular sizes. This morphofunctional discrepancy may be partly explained by the difference of tissue preparation methods, because we frequently detected the modified immunoreactivity of serum proteins within some blood vessels of ovarian tissues prepared by IM-DH or PF-DH. The components and structures of intercellular communication between vascular endothelial cells of ovaries, which regulate the limited passage of larger molecules, would be revealed at an electron microscopic level in the future studies with IVCT.

On the other hand, it is generally accepted that the basement membrane of blood vessels in some animal organs can act as a molecular sieve with pore sizes determined by the charge and spatial arrangement of its components, such as type IV collagen, laminin, and heparan sulfate proteoglycans (Holmquist et al. 2004, Farquhar 2006). In the previous studies, it was already shown that certain basement membranes around blood vessels prevented leakage of large serum proteins (Ohlson et al. 2000, Maina \& West 2005). At the glomerular capillary loops of kidneys, the basement membranes prevented filtration of certain serum proteins for urine production. They also permitted gaseous diffusion without passage of serum proteins through the blood capillaries of animal lungs. Our findings indicated a molecular sieve function of follicular basement membranes in living mouse ovaries, which would regulate some metabolic exchanges between the inside and outside of the ovarian follicles during their development. Indeed, it was already reported that the follicular basement membrane might seal the extracellular matrix in the developing ovarian follicles during their rapid volume expansion (Irving-Rodgers \& Rodgers 2000). However, in the present study, the permselectivity of follicular basement membranes was found to be different from that around the blood vessels. The functional difference may be attributed to their distinctive components. As the basement membrane with different components exhibits more complicated ultrastructural features (Takami et al. 1991, Xu \& Ling 1994), the follicular basement membrane is a specific structure that has to be dramatically changed during the follicular development and ovulation (Zamboni 1974, Rodgers et al. 2003). Our finding that the BFB was functioning at an early stage of folliculogenesis also supports the significant involvement of basement membranes as the BFB, which are continuously 
enclosing the ovarian follicles at all development stages, although some of their components would be changed at different stages (Rodgers et al. 1999).

It was previously reported that there was no $|\alpha|$ in mouse follicular fluid (Hess et al. 1998), although our findings suggest its presence in the mouse follicles. This discrepancy may be due to the difference in probes used and experimental procedures, because Hess et al. used fluorescence-labeled extrinsic proteins that were injected $3 \mathrm{~h}$ prior to fixation; the diffusion of $|\alpha|$ from blood vessels into the follicles, which would be driven by the osmotic potential of large glycosaminoglycans and proteoglycans (Clarke et al. 2006), may take longer time in living mouse ovaries. Another possibility would be the difference of tissue preparation methods, because it is sometimes difficult to visualize the in situ distribution of serum soluble molecules with the conventional chemical fixation. Actually, we failed in our immunohistochemical visualization of $|\alpha|$ in the tissue specimens prepared with the common IM (data not shown). Other reports on porcine and bovine ovaries also suggested the presence of $|\alpha|$ in their follicles, supporting our immunohistochemical findings (Nagyova et al. 2004, Clarke et al. 2006).

In conclusion, the morphology of living mouse ovaries and immunolocalization of soluble serum proteins were precisely revealed by the IVCT, as compared with the conventional preparation methods. The different immunolocalizations of serum proteins represent the size selectivity at two different sites of BFB, and the follicular basement membrane would be responsible for the permeability of serum proteins with intermediate molecular sizes, whereas the vascular endothelial cells would be involved in the permselectivity of those with larger molecular sizes.

\section{Acknowledgements}

The authors declare that there is no conflict of interest that would prejudice the impartiality of this scientific work.

\section{References}

Aijaz S, Balda MS \& Matter K 2006 Tight junctions: molecular architecture and function. International Review of Cytology $\mathbf{2 4 8}$ 261-298.

Andersen MM, Kroll J, Byskov AG \& Faber M 1976 Protein composition in the fluid of individual bovine follicles. Journal of Reproduction and Fertility 48 109-118.

Angelucci S, Ciavardelli D, Di Giuseppe F, Eleuterio E, Sulpizio M, Tiboni GM, Giampietro F, Palumbo P \& Di Ilio C 2006 Proteome analysis of human follicular fluid. Biochimica et Biophysica Acta 1764 1775-1785.

Bazzoni G 2006 Endothelial tight junctions: permeable barriers of the vessel wall. Thrombosis and Haemostasis 95 36-42.
Bazzoni G \& Dejana E 2004 Endothelial cell-to-cell junctions: molecular organization and role in vascular homeostasis. Physiological Reviews 84 869-901.

Clarke HG, Hope SA, Byers S \& Rodgers RJ 2006 Formation of ovarian follicular fluid may be due to the osmotic potential of large glycosaminoglycans and proteoglycans. Reproduction 132 119-131.

Collins A, Palmer E, Bezard J, Burke J, Duchamp G \& Buckley T 1997 A comparison of the biochemical composition of equine follicular fluid and serum at four different stages of the follicular cycle. Equine Veterinary Journal Supplement 25 12-16.

Cran DG, Moor RM \& Hay MF 1976 Permeability of ovarian follicles to electron-dense macromolecules. Acta Endocrinologica 82 631-636.

Donahue RP \& Stern S 1968 Follicular cell support of oocyte maturation: production of pyruvate in vitro. Journal of Reproduction and Fertility 17 395-398.

Dvorak HF, Brown LF, Detmar M \& Dvorak AM 1995 Vascular permeability factor/vascular endothelial growth factor, microvascular hyperpermeability, and angiogenesis. American Journal of Pathology 146 1029-1039.

Farquhar MG 2006 The glomerular basement membrane: not gone, just forgotten. Journal of Clinical Investigation 116 2090-2093.

Gartner JP \& Hiatt JL 1994 Female reproductive system. In Color Atlas of Histology, 2 edn, pp 318-324. Eds JP Gartner \& JL Neill. Baltimore: William \& Wilkins.

Hess KA, Chen L \& Larsen WJ 1998 The ovarian blood follicle barrier is both charge- and size-selective in mice. Biology of Reproduction $\mathbf{5 8}$ 705-711.

Hippe-Sanwald S 1993 Impact of freeze substitution on biological electron microscopy. Microscopy Research and Technique $\mathbf{2 4}$ 400-422.

Holmquist P, Sjoblad S \& Torffvit O 2004 Pore size and charge selectivity of the glomerular membrane at the time of diagnosis of diabetes. Pediatric Nephrology 19 1361-1366.

Irving-Rodgers HF \& Rodgers RJ 2000 Ultrastructure of the basal lamina of bovine ovarian follicles and its relationship to the membrana granulosa. Journal of Reproduction and Fertility 118 221-228.

Jiang JY, Macchiarelli G, Miyabayashi K \& Sato E 2002 Follicular microvasculature in the porcine ovary. Cell and Tissue Research $\mathbf{3 1 0}$ 93-101.

Kanzaki H, Okamura H, Okuda Y, Takenaka A, Morimoto K \& Nishimura T 1982 Scanning electron microscopic study of rabbit ovarian follicle microvasculature using resin injection-corrosion casts. Journal of Anatomy 134 697-704.

Kellenberger E 1991 The potential of cryofixation and freeze substitution: observations and theoretical considerations. Journal of Microscopy 161 183-203.

Kitai H, Yoshimura Y, Wright KH, Santulli R \& Wallach EE 1985 Microvasculature of preovulatory follicles: comparison of in situ and in vitro perfused rabbit ovaries following stimulation of ovulation. American Journal of Obstetrics and Gynecology 152 889-895.

Kranzfelder D \& Maurer-Schultze B 1989 Development of the perifollicular capillary network. Autoradiographic and morphometric studies in the rabbit ovary. European Journal of Obstetrics, Gynecology, and Reproductive Biology 30 163-171.

Li Z, Terada N, Ohno N \& Ohno S 2005 Immunohistochemical analyses on albumin and immunoglobulin in acute hypertensive mouse kidneys by 'in vivo cryotechnique'. Histology and Histopathology 20 807-816.

Li Z, Ohno N, Terada N \& Ohno S 2006a Immunolocalization of serum proteins in living mouse glomeruli under various hemodynamic conditions by 'in vivo cryotechnique'. Histochemistry and Cell Biology 126 399-406.

Li Z, Ohno N, Terada N, Zhou D, Yoshimura A \& Ohno S 2006b Application of periodic acid-Schiff fluorescence emission for immunohistochemistry of living mouse renal glomeruli by an 'in vivo cryotechnique'. Archives of Histology and Cytology 69 147-161. 
Liao X, Terada N, Ohno N, Li Z, Fujii Y, Baba T \& Ohno S 2006 Immunohistochemical study of serum albumin in normal and cadmium-treated mouse testis organs by 'in vivo cryotechnique'. Histology and Histopathology 21 35-40.

Macchiarelli G 2000 The microvasculature of the ovary: a review by S.E.M. of vascular corrosion casts. Journal of Reproduction and Development 46 207-225.

Madara JL 1989 Loosening tight junctions. Lessons from the intestine. Journal of Clinical Investigation 83 1089-1094.

Maina JN \& West JB 2005 Thin and strong! The bioengineering dilemma in the structural and functional design of the blood-gas barrier. Physiological Reviews 85 811-844.

Nagyova E, Camaioni A, Prochazka R \& Salustri A 2004 Covalent transfer of heavy chains of inter- $\alpha$-trypsin inhibitor family proteins to hyaluronan in in vivo and in vitro expanded porcine oocyte-cumulus complexes. Biology of Reproduction 71 1838-1843.

Ohlson M, Sorensson J \& Haraldsson B 2000 Glomerular size and charge selectivity in the rat as revealed by FITC-ficoll and albumin. American Journal of Physiology. Renal Physiology 279 F84-F91.

Ohno S, Terada N, Fujii Y, Ueda H \& Takayama I 1996 Dynamic structure of glomerular capillary loop as revealed by an in vivo cryotechnique. Virchows Archiv 427 519-527.

Ohno N, Terada N \& Ohno S 2004 Advanced application of the in vivo cryotechnique to immunohistochemistry for animal organs. Acta Histochemica et Cytochemica 37 357-364.

Ohno N, Terada N, Murata S, Katoh R \& Ohno S 2005a Application of cryotechniques with freeze-substitution for the immunohistochemical demonstration of intranuclear PCREB and chromosome territory. Journal of Histochemistry and Cytochemistry 53 55-62.

Ohno N, Terada N, Tanaka J, Yokoyama A, Yamakawa H, Fujii Y, Baba T, Ohara O \& Ohno S 2005b Protein 4.1 G localizes in rodent microglia. Histochemistry and Cell Biology 124 477-486.

Ohno N, Terada N \& Ohno S 2006 Histochemical analyses of living mouse liver under different hemodynamic conditions by 'in vivo cryotechnique'. Histochemistry and Cell Biology 126 389-398.

Orlova VV, Economopoulou M, Lupu F, Santoso S \& Chavakis T 2006 Junctional adhesion molecule-C regulates vascular endothelial permeability by modulating VE-cadherin-mediated cell-cell contacts. Journal of Experimental Medicine 203 2703-2714.

Powers RW, Chen L, Russell PT \& Larsen WJ 1995 Gonadotropinstimulated regulation of blood-follicle barrier is mediated by nitric oxide. American Journal of Physiology 269 E290-E298.

Rodgers RJ, Lavranos TC, van Wezel IL \& Irving-Rodgers HF 1999 Development of the ovarian follicular epithelium. Molecular and Cellular Endocrinology 151 171-179.

Rodgers RJ, Irving-Rodgers HF \& Russell DL 2003 Extracellular matrix of the developing ovarian follicle. Reproduction 126 415-424.

Ross MH, Reith EJ \& Romrell LJ 1989 Female reproductive system. In Histology: A Text and Atlas, 2 edn, pp 649-655. Eds MH Ross, EJ Reith \& LJ Romrell. Baltimore: Williams \& Wilkins.
Shalgi R, Kraicer P, Rimon A, Pinto M \& Soferman N 1973 Proteins of human follicular fluid: the blood-follicle barrier. Fertility and Sterility 24 429-434.

Shiurba R 2001 Freeze-substitution: origins and applications. International Review of Cytology 206 45-96.

Takami H, Naramoto A, Shigematsu H \& Ohno S 1991 Ultrastructure of glomerular basement membrane by quick-freeze and deep-etch methods. Kidney International 39 659-664.

Terada N, Ohno N, Li Z, Fujii Y, Baba T \& Ohno S 2005 Detection of injected fluorescence-conjugated IgG in living mouse organs using 'in vivo cryotechnique' with freeze-substitution. Microscopy Research and Technique 66 173-178.

Terada N, Ohno N, Li Z, Fujii Y, Baba T \& Ohno S 2006a Application of in vivo cryotechnique to the examination of cells and tissues in living animal organs. Histology and Histopathology 21 265-272.

Terada N, Ohno N, Ohguro H, Li Z \& Ohno S 2006 b Immunohistochemical detection of phosphorylated rhodopsin in light-exposed retina of living mouse with in vivo cryotechnique. Journal of Histochemistry and Cytochemistry 54 479-486.

Wolburg H \& Lippoldt A 2002 Tight junctions of the blood-brain barrier: development, composition and regulation. Vascular Pharmacology 38 323-337.

Xu J \& Ling EA 1994 Studies of the ultrastructure and permeability of the blood-brain barrier in the developing corpus callosum in postnatal rat brain using electron dense tracers. Journal of Anatomy $\mathbf{1 8 4}$ 227-237.

Yamada O, Abe M, Takehana K, Iwasa K \& Hiraga T 1994 Scanning electron microscopical observation of the intramitochondrial body in the bovine corpus luteum during pregnancy and after parturition. Journal of Veterinary Medical Science 56 459-464.

Zachariae F 1958 Studies on the mechanism of ovulation: permeability of the blood-liquor barrier. Acta Endocrinologica 27 339-342.

Zamboni L 1974 Fine morphology of the follicle wall and follicle celloocyte association. Biology of Reproduction 10 125-149.

Zea-Aragon Z, Terada N, Ohno N, Fujii Y, Baba T \& Ohno S 2004 Effects of anoxia on serum immunoglobulin and albumin leakage through blood-brain barrier in mouse cerebellum as revealed by cryotechniques. Journal of Neuroscience Methods 138 89-95.

Zhuo L \& Kimata K 2001 Cumulus oophorus extracellular matrix: its construction and regulation. Cell Structure and Function $\mathbf{2 6}$ 189-196.

Zhou D, Ohno N, Terada N, Li Z, Morita H, Inui K, Yoshimura A \& Ohno S 2007 Immunohistochemical analyses on serum proteins in nephrons of protein-overload mice by 'in vivo cryotechnique'. Histology and Histopathology 22 137-145.

Received 6 February 2007

First decision 15 March 2007

Accepted 3 May 2007 